\title{
Design of Bionic Bucket Teeth and Drag Reduction Analysis
}

\author{
Zhifeng Zhang ${ }^{1,2, a}$, Yan Zhang ${ }^{1,2, b^{*}}$, Yueying Zhu ${ }^{1,2, c}$, Junxia Zhang ${ }^{1,2, d}$ \\ ${ }^{1}$ Tianjin Key Laboratory of Integrated Design and On-line Monitoring for Light Industry \& Food \\ Machinery and Equipment, Tianjin 300222, P. R. China \\ ${ }^{2}$ College of Mechanical Engineering, Tianjin University of Science and Technology, Tianjin 300222 , \\ P. R. China

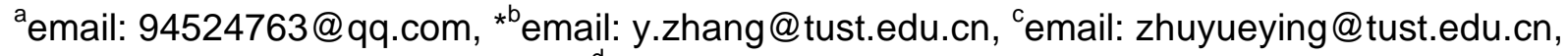 \\ demail: zjx@tust.edu.cn
}

Keywords: bionics, bucket teeth, wedging, mole cricket

Abstract. Bucket teeth are located at the end of a bucket, which enable the bucket to dig into soil with a lower resistance. In this paper, the configuration information of a claw tooth of mole crickets was extracted and used for designing the configuration of a bionic bucket tooth which was modified from the CAT-320D excavator bucket tooth. The soil wedging processes of the bionic bucket tooth and the CAT-320D bucket tooth were analyzed using finite element analysis, and the soil wedging performances of the two teeth were compared. The results show that the concave side of the bionic bucket tooth is easy to make a large deformation of the soil, which is help to break the soil interface. The soil disturbance on the convex side of the bionic bucket tooth is small, which is help to reduce the compaction of the dorsal soil and friction. Compared with the CAT-320D bucket tooth, the bionic bucket tooth has a greater effect on wedging the soil, in which the total stress of the soil is smaller. The analysis on the total soil energy shows that the energy consuming of the bionic bucket tooth is lower than that of the CAT-320D bucket tooth.

\section{Introduction}

The excavator is consisted by bucket, bucket teeth, tooth holder etc, and the bucket is an important part of an excavator. Bucket teeth are located at the end of a bucket, and wedge into soil with a certain angle during operation, which enable the bucket to dig into soil with a lower resistance. The common methods for improving the digging performance of bucket teeth mainly include configuration modification[1,2], wear resistance enhancing[3,4], and excavation trajectory optimization[5]. The geometric configuration of a bucket tooth directly affects the soil wedging performance[6,7]. Therefore, the optimization of the bucket teeth configuration, not only can effectively reduce the wedging resistance, but also can reduce wear of bucket teeth. The engineering bionics provides a new insight to improving or optimizing the engineering design by analyzing the basic principles of the outstanding biological functions of animals or plants[8]. For example, as a typical soil animal, mole crickets possess excellent excavation performance with shovel-like forelimbs highly developed for burrowing. The claw teeth of mole crickets can be used as the bionic prototype of new type bionic bucket teeth[9].

In this paper, the configuration information of a claw tooth of mole crickets was extracted and used for designing the configuration of the bionic bucket tooth which was modified from the CAT-320D excavator bucket tooth. The soil wedging processes of the bionic and the CAT-320D bucket tooth were analyzed using finite element analysis, and the soil wedging performances of the two bucket teeth were compared.

\section{Materials and methods}

Bionic bucket tooth design. The first claw tooth of mole crickets (Gryllotalpa orientalis) was chose as the bionic prototype. The claw tooth was prepared with epoxy embedding and sliced. The axial and radial cross sections were obtained by layer-by-layer grinding. The cross section images were captured 
using a stereoscopic microscope. After that, the images were imported into the AutoCAD software and the contour lines of the claw tooth in each section were fitted by multi-segment tangent arc fitting. The data for fitting are listed in Tables 1 and 2, and the fitted curves are shown in Figs. 1 and 2.

Table 1 Fitting curve data in axial direction

\begin{tabular}{cccccccc}
\hline & \multicolumn{3}{c}{ Upper line } & \multicolumn{4}{c}{ Lower line } \\
\cline { 2 - 8 } & 1 & 2 & 3 & 1 & 2 & 3 & 4 \\
\hline Start points & $(0$, & $(260.00$, & $(400.00$, & $(0$, & $(255.00$, & $(285.00$, & $(375.00$, \\
$(\mathrm{mm})$ & $20.00)$ & $290.00)$ & $320.00)$ & $-65.00)$ & $180.00)$ & $235.00)$ & $300.00)$ \\
End points & $(260.00$, & $(400.00$, & $(450.00$, & $(255.00$, & $(285.00$, & $(375.00$, & $(420.00$, \\
$(\mathrm{mm})$ & $290.00)$ & $320.00)$ & $310.00)$ & $180.00)$ & $235.00)$ & $300.00)$ & $305.00)$ \\
Circle center & $(555.70$, & $(388.88$, & $(386.10$, & $(-128.78$, & $(452.30$, & $(408.42$, & $(484.70$, \\
$(\mathrm{mm})$ & $-254.93)$ & $30.21)$ & $120.48)$ & $324.25)$ & $108.07)$ & $158.90)$ & $-482.34)$ \\
Radius $(\mathrm{mm})$ & 620.75 & 202.45 & 289.90 & 412.40 & 213.75 & 143.23 & 793.02 \\
\hline
\end{tabular}

Table 2 Fitting curve data in radial direction

\begin{tabular}{ccccccccc}
\hline & \multicolumn{3}{c}{ Upper line } & \multicolumn{5}{c}{ Lower line } \\
\cline { 2 - 9 } & 1 & 2 & 3 & 1 & 2 & 3 & 4 & 5 \\
\hline Start points & $(2.57$, & $(41.20$, & $(69.10$, & $(4.67$, & $(17.30$, & $(40.67$, & $(69.63$, & $(93.00$, \\
$(\mathrm{mm})$ & $4.22)$ & $38.50)$ & $38.50)$ & $-1.63)$ & $-1.03)$ & $-0.82)$ & $-0.82)$ & $-1.03)$ \\
End points & $(41.20$, & $(69.10$, & $(107.73$, & $(17.30$, & $(40.67$, & $(69.63$, & $(93.00$, & $(105.63$, \\
$(\mathrm{mm})$ & $38.50)$ & $38.50)$ & $4.22)$ & $-1.03)$ & $-0.82)$ & $-0.82)$ & $-1.03)$ & $-1.63)$ \\
Circle center & $(164.41$, & $(55.15$, & $(-54.11$, & $(18.60$, & $(27.99$, & $(55.15$, & $(82.31$, & $(91.70$, \\
$\quad(m m)$ & $-139.23)$ & $21.49)$ & $-139.23)$ & $-160.70)$ & $110.35)$ & $-171.65)$ & $110.35)$ & $-160.70)$ \\
Radius (mm) & 216.26 & 22.00 & 216.26 & 159.68 & 111.89 & 171.44 & 111.89 & 159.68 \\
\hline
\end{tabular}

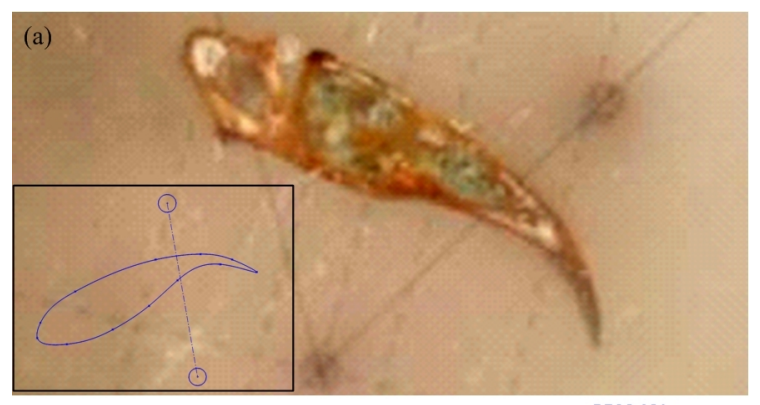

(b)

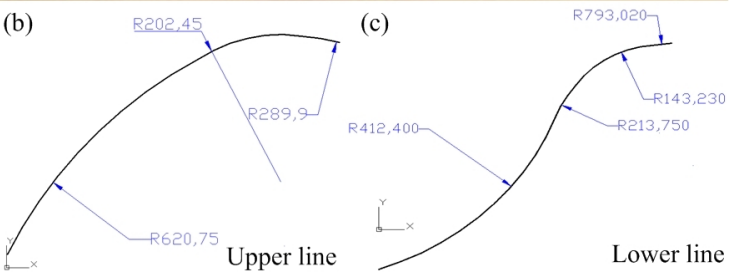

Fig. 1 Contour fitting in axial direction
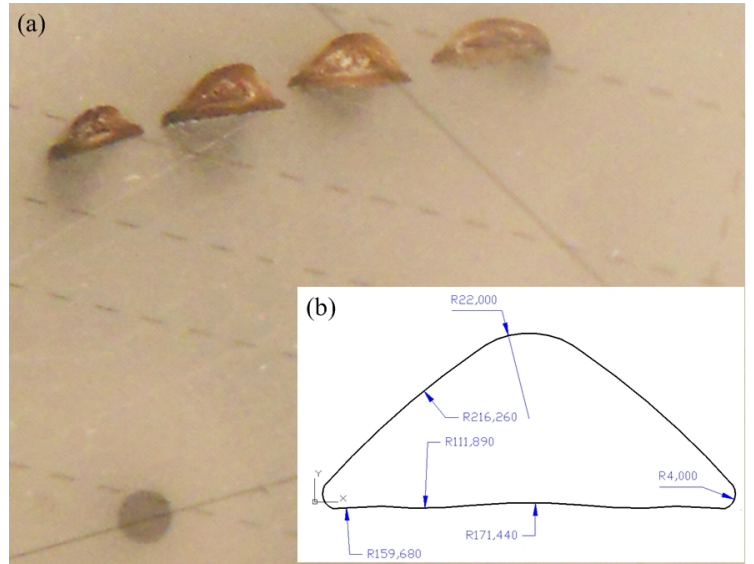

Fig. 2 Contour fitting in radial direction

The radial and axial fitting contours of each section were imported into the Solidworks software and enlarged to fit the geometric dimension of the CAT-320D excavator bucket. The slice spacing between two sections was also enlarged. The solid model of the bionic bucket tooth was established using "loft" command. As a consequence, the geometrical configuration of the bionic bucket tooth model had the biological characteristics of the mole cricket claw tooth, as shown in Fig. 3.

Numerical simulation. The nonlinear dynamic finite element analysis software ANSYS/LS-DYNA was used to compare the soil wedging performance of the CAT-320D and the bionic bucket tooth. The SOLID164 unit was selected for soil and the bucket teeth. Rigid material model was used for bucket teeth and plastic follow-up material model was used for soil[10]. Soil model was cubic solid with the size of $400 \times 300 \times 200$. The single point integral and Lagrange algorithm were applied in the simulation. 
The wedging angle was $90^{\circ}$. The erosion type was face contact i.e. ESTS - Eroding type. The wedging speed was $0.5 \mathrm{~m} / \mathrm{s}$, which is the common operating speed of excavator buckets. The parameters of the soil model and the bucket tooth model were listed in Table 3 and 4.

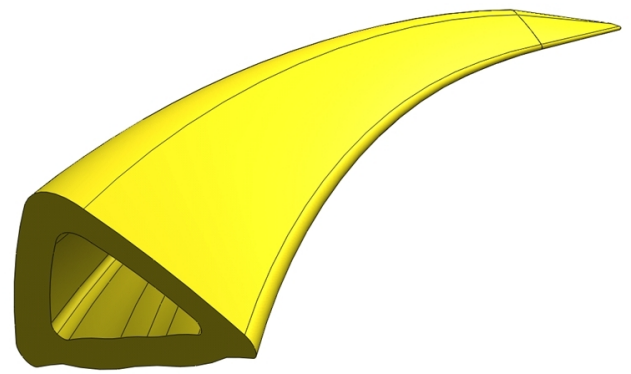

Fig. 3 3D model of the bionic bucket tooth

Table 3 Material parameters of soil model

\begin{tabular}{ccccccccc}
\hline $\begin{array}{c}\text { Density } \\
{\left[\mathrm{kg} / \mathrm{mm}^{3}\right]}\end{array}$ & $\begin{array}{c}\text { Elastic } \\
\text { modulus }[\mathrm{kPa}]\end{array}$ & \multicolumn{2}{c}{ Poisson's Yield stress } & Tangent & Hardening & \multicolumn{2}{c}{$\begin{array}{c}\text { Strain } \\
\text { ratio }\end{array}$} & $\begin{array}{c}\text { Strain Failure } \\
\text { modulus }[\mathrm{kPa}]\end{array}$ \\
\hline $1.910 \times 10^{-6}$ & $1.2 \times 10^{3}$ & 0.38 & $8 \mathrm{e} 2$ & $1 \times 10^{2}$ & 0 & 50 & 4 & 0.8 \\
\hline
\end{tabular}

Table 4 Material parameters of bucket teeth model

\begin{tabular}{ccc}
\hline Density $\left[\mathrm{kg} / \mathrm{mm}^{3}\right]$ & Elastic modulus $[\mathrm{kPa}]$ & Poisson's ratio \\
\hline $7.830 \times 10^{-6}$ & $2.07 \times 10^{3}$ & 0.3 \\
\hline
\end{tabular}

\section{Results}

Drag reduction effect of the bionic bucket tooth. (1) Stress distribution analysis. Fig. 4 shows the stress distribution of the soil after wedging about $0.9 \mathrm{~s}$. In the horizontal direction as shown in the figure, the soil disturbance on both sides of the CAT-320D bucket tooth is symmetrical distribution while a larger soil disturbance is found at the concave side and a smaller soil disturbance is found at the convex side of the bionic bucket tooth when the wedging depths of the two teeth are same. On one hand, the larger deformation at the concave side of the bionic bucket tooth helps to produce particle breakage of soil mass at the wedging zone. On the other hand, the smaller soil disturbance at the convex side can cause smaller soil compacting effect of the dorsal soil, which helps to reduce the friction. In the vertical direction, the mean stress of the soil caused by the bionic bucket tooth is slight smaller than that by the CAT-320D bucket tooth. During the wedging process, the smaller the mean stress of the soil in the axial direction, the smaller the compaction of the beneath soil, the smaller the resistance breaking the soil interface. In addition, it can be seen from Fig. 4(a) that the stress of the soil in contact with the side wall of the CAT-320D bucket tooth is greater than that of the bionic bucket tooth at the soil surface layer. The greater the stress, the larger friction of the tooth at the side wall is.

(2) Stress at the wedging contact point. Fig. 5 shows the stress curves of soil at the wedging contact point between the bucket tooth and the soil unit. The peak value presents the maximum stress of the soil unit when a bucket tooth begins wedging into soil. After that, this value will drop to zero due to the failure of the soil unit at the contact point. It can be seen from the figure that the bionic bucket tooth can produce greater pressure to the soil unit at the contact point than the CAT-320D bucket tooth. It means that the damage effect of the bionic bucket tooth to the soil interface is larger than that of the CAT-320D bucket tooth.

(3) Soil total energy analysis. In the interaction between the bucket tooth model and the soil model, the total energy of soil includes soil kinetic energy and soil energy. The total energy totally comes from the bucket tooth model. As shown in Fig. 6, it can be obtained that the soil model interacting with the bionic bucket tooth obtains less energy than the CAT-320D bucket tooth under the same conditions. It means that in a same wedging process, the changes of kinetic energy of the bionic bucket tooth are relatively small. According to the formula of kinetic energy $0.5 m v^{2}$, i.e. the kinetic energy of an object 
is proportional to the square of its velocity. The small of kinetic energy variation represents that the amount of change in speed is relatively small. Therefore, energy consumption of the bionic bucket tooth in the soil wedging process is lower than that of the CAT-320D bucket tooth in a same circumstance.

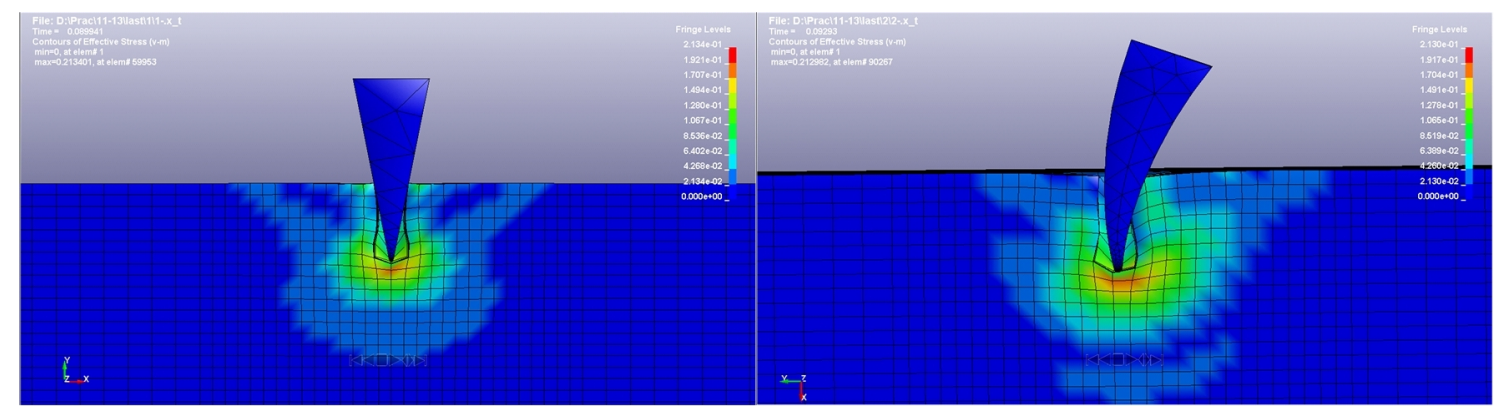

(a) CAT-320D bucket tooth

(b) Bionic bucket tooth

Fig. 4 Simulation of wedging process of the CAT-320D and bionic bucket tooth

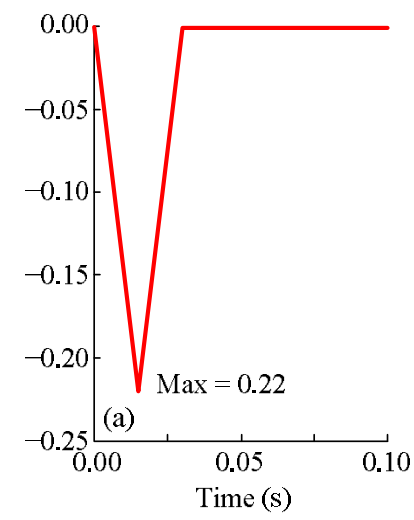

(a) CAT-320D bucket tooth

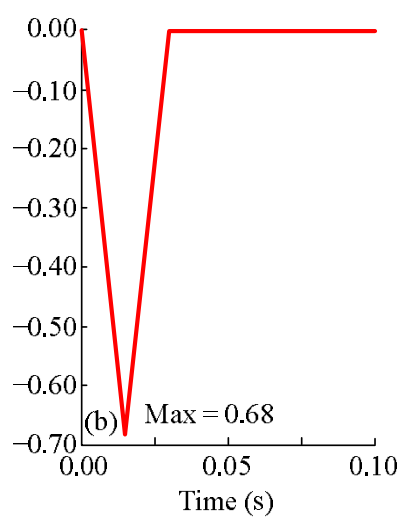

(b) Bionic bucket tooth in soil contact unit

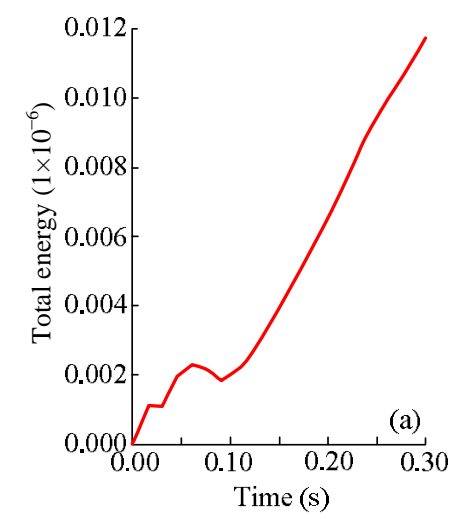

(a) CAT-320D bucket tooth

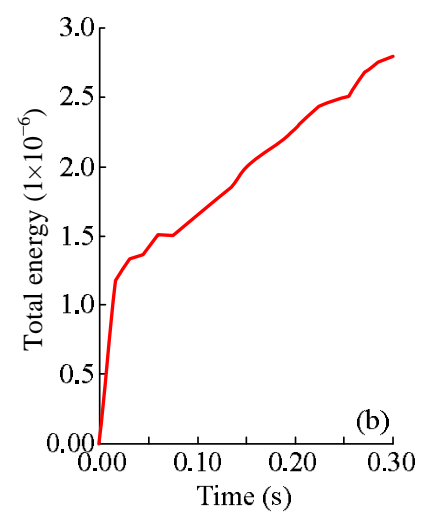

(b) Bionic bucket tooth

Fig. 6 Total soil energy in soil wedging

Bionic excavator bucket design. The design of the bionic excavator bucket is based on the CAT-320D excavator bucket[11]. The 2D bionic structural model of the bucket main body is designed by using the fitting curve obtained from the axial cross section of the mole cricket claw tooth. The features of the structural model of the bucket main model are shown in Fig. 7. Other components, includes tooth holder, tooth plate and bucket floor are also designed to make the lower surface of the bucket to form a smooth curved surface. While the shape of side plate, wall panels and edge plate on two sides of bucket were also designed according to the curved surface of the lower surface. 


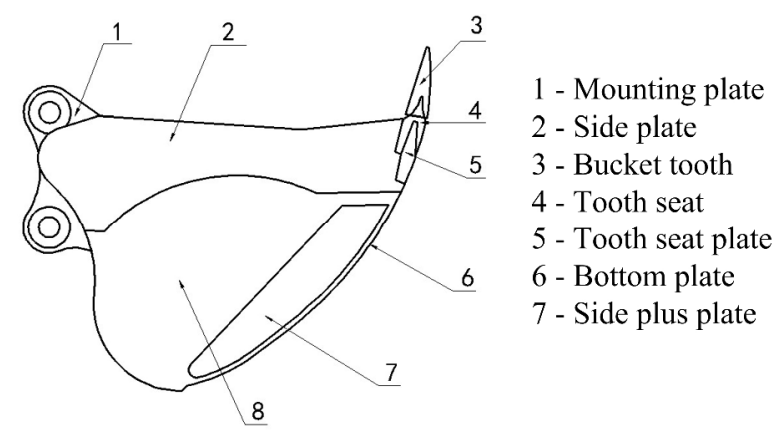

Fig. 7 Bionic excavator bucket structure

\section{Conclusions}

Based on a CAT-320D excavator bucket tooth, a bionic excavator bucket tooth was designed by using the axial and radial cross-section contours of the mole cricket claw tooth. The stress distribution of the soil and the stress level at the wedging contact point were analyzed and compared between the bionic bucket tooth and the CAT-320D bucket tooth using simulation methods. The results show that the concave side of the bionic bucket tooth is easy to make a large deformation of the soil which is help to break the soil interface. The soil disturbance on the convex side of the bionic bucket tooth is small, which is help to reduce the compaction of the dorsal soil and friction. Compared with the CAT-320D bucket tooth, the bionic bucket tooth has a greater effect on wedging soil, in which the total stress of the soil is smaller. It means that the compaction of the soil is small, thus reducing the excavation resistance, so that the bucket tooth is much easier to wedging into the soil. The analysis on the total soil energy shows that the energy consuming of the bionic bucket tooth is lower than that of the CAT-320D bucket tooth. Moreover, the matching bucket of the bionic bucket teeth was designed to realize a smooth lower curved surface of the bucket.

\section{Acknowledgements}

This work was financially supported by the National Natural Science Foundation of China (51405341 and 51505332) and the Tianjin Research Program of Application Foundation and Advanced Technology (15JCYBJC19300 and 15JCQNJC06900).

\section{References}

[1] B.S. Wang: Mining. Process. Equip. (2000), p. 31

[2] Z.L. Chen and Y.L. Li: Mining. Process. Equip. (1992), p. 9-11

[3] W.B. Wang: Constr. Mach. Equip. (1989), p. 48-50

[4] J.E. Fernandez, R. Vijande, R. Tucho, J. RodríGuez and A. Martin: Wear Vol. 250 (2001), p. $11-18$

[5] J. Maciejewski and A. Jarzebowski: J. Terramechanics Vol. 39 (2002), p. 161-179

[6] D. Musielski and W. Keska: J. Res. Appli. Agric. Eng. Vol. 52 (2007), p. 27-29

[7] D.C. Brooker: Int. J. Press. Vessels Piping Vol. 82 (2005), p. 825-832

[8] L.Q. Ren and Y.H. Liang: Sci. China E Vol. 52 (2009), p. 2791-2800

[9] L.Q. Ren: Sci. China E Vol. 52 (2009), p. 273-284

[10] A.M. Mouazen and M. Nemenyi: J. Agric. Eng. Res. Vol. 72 (1999), p. 37-51

[11] JG/T 90-1999. Technical Requirements for Teeth of Hydraulic Excavators (China Standard Press, Beijing, 1999). 